\title{
Hipotiroidismo: implicaciones clínicas y económicas en un área de salud
}

\author{
D. DE LUIS ROMÁN, J. L. GONZÁLEZ PELÁEZ, R. ALLER, M. GONZÁLEZ \\ SAGREDO, L. CUELLAR, M. C. TERROBA
}

Unidad de Endocrinología y Nutrición Clínica. Unidad de Apoyo a la Investigación. Hospital del Río Hortega. Instituto de Endocrinología y Nutrición. Facultad de Medicina. Valladolid

\author{
HYPOTHYROIDISM: CLINICAL AND ECONOMICAL IMPLICATIONS \\ IN A HEALTH AREA
}

\section{RESUMEN}

Objetivo: Analizar las características epidemiológicas, bioquímicas y clínicas al inicio del diagnóstico de hipotiroidismo, la dosis de hormona tiroidea necesaria para un correcto control, así como los costes directos generados anualmente, todo ello referido a pacientes con hipotiroidismo atendidos en un área de salud.

Materiales y métodos: Se revisan las historias clínicas correspondientes a 75 pacientes diagnosticados de hipotiroidismo y atendidos en la consulta de Endocrinología de un área de salud, agrupando los datos recogidos en tres categorías: epidemiológicos, bioquímicos y clínicos, y económicos.

Resultados: Del total de pacientes estudiados, el 94,7\% eran mujeres y el 5,3\% hombres, con una edad media de 52,07 $\pm 16,58$ años. La patología responsable del hipotiroidismo más frecuente fue la autoinmune, incluyendo la tiroiditis de Hashimoto $(48,0 \%)$ y la tiroiditis autoinmune atrófica $(46,6 \%)$. El valor medio de la TSH $(\mathrm{mUI} / \mathrm{ml})$ fue de $17,57 \pm$ 40,14 , y el de los anticuerpos antitiroideos $(\mathrm{UI} / \mathrm{ml})$ fue de $629,30 \pm$ $1.049,14$ para los ACTPO y de $532,11 \pm 1.028,34$ para los ACTG. Un $58,7 \%$ de los pacientes presentaba bocio, pero sólo en el 2,6\% era en un grado evidente. La dosis media de tratamiento sustitutivo con T4 fue de $81,00 \pm 53,56 \mathrm{mg} / \mathrm{d}$ á. La media de visitas anuales a la consulta de Endocrinología hasta el correcto control de la enfermedad fue de 2,67 $\pm 1,08$. El mayor coste generado por estos pacientes fue el de la consulta especializada, con una media $(€ /$ año) de $160 \pm 65 €$ y un coste total medio (consulta, analíticas y dosis sustitutiva T4) (€/año) de $12,9 \pm 81,5 €$. Comparando las dos patologías autoinmunes más prevalentes, observamos un mayor nivel de ACTPO en los pacientes con tiroiditis de Hashimoto, con un mayor número de visitas a la consulta y un mayor coste en tratamiento sustitutivo con $\mathrm{T} 4$, resultando en definitiva un mayor coste total en dichos pacientes.

Conclusiones: La patología más prevalente es la autoinmune (incluyendo la T. de Hashimoto y la tiroiditis autoinmune atrófica), con mayores niveles de ACTPO en la tiroiditis de Hashimoto. El coste total es superior en los pacientes con tiroiditis de Hashimoto.

PALABRAS CLAVE: Hipotiroidismo. Diagnóstico. Costes. Tiroiditis autoinmune.

\section{ABSTRACT}

Objective: To analize the clinical, biochemical and epidemiological characteristics in patients with hypothyroidism, substitutive dose and anual direct costs, in a health area.

Material and methods: Seventy five patients attended in a health area were included in the review. Data was divided in epidemiological, bio chemical, clinics and economics.

Results: A $94.7 \%$ were females and $5.3 \%$ males, with an average age of $52.07+16.5$ years. The most frequent disease was Thyroiditis of Has himoto $48 \%$ and athrophic thyroiditis $46.6 \%$. Average value of TSH was $17.57+40.8 \mathrm{mUI} / \mathrm{ml}$ and TPO 629.3+1049 UI/ml and Ac TG 532.1+1028 UI/ml. A $58,7 \%$ of patients had goiter, only envident $2.6 \%$. The average dose of T4 was $81+53.5 \mathrm{mg} /$ day. The average number of visits was $2.67+1.08$. The highest cost was due to specialists visit $160+65$ euros with a total average cost of 212.9+81.5 euros. Levels of TPO was higher in T. Hashimoto, with a higher number of visits and costs.

Conclusions: The most prevalent disease was autoimmune (T Hashi moto and Athrophic thyroiditis) with higher levels of TPO in Hashimoto. Total cost is higher in T. Hashimoto.

KEY WORDS: Hypothyroidism. Diagnosis. Costs. Autoimmune athrop hic thyroiditis.

De Luis Román D, González Peláez JL, Aller R, González Sagredo M, Cuellar L, Terroba MC. Hipotiroidismo: implicaciones clínicas y eco nómicas en un área de salud. An Med Interna (Madrid) 2003; 20: 127-133.

\section{INTRODUCCIÓN}

El hipotiroidismo representa un cuadro clínico complejo definido analíticamente por unos niveles de TSH elevados y
T4 libre disminuida y clínicamente por la sintomatología derivada de la falta de actividad de las hormonas tiroideas a nivel de los tejidos. Por otra parte hay que distinguir el concepto de hipotiroidismo subclínico, que ocurre en individuos asintomá-

Trabajo aceptado: 14 de mayo de 2002

Correspondencia: Daniel de Luis. Director del Instituto Endocrinología y Nutrición. c/. Los Perales, 16.47130 Valladolid.dadluis@yahoo.es. 
ticos y se caracteriza por el hallazgo de cifras elevadas de TSH con niveles normales de T3 y T4 totales y libres (1).

En razón de la definición de hipotiroidismo, cuyos solapados e inespecíficos signos y síntomas pueden ser equivocadamente atribuidos a otras enfermedades, la prevalencia es muy variable según los estudios (0,6 a 7\%) (2). Como cifras más aceptadas se habla de una prevalencia de $0,1 \%$ en hombres $y$ de 1,5\% en mujeres (3). En cuanto al hipotiroidismo subclínico, la prevalencia oscila entre el 2 y el $8 \%$ de la población general (4). En el estudio Whickham (5) el $8 \%$ de mujeres y el $3 \%$ de hombres tenían hipotiroidismo subclínico. Si incluimos ambas entidades podríamos llegar a una afectación del 5,4\% de la población general. Además, un hecho sobradamente conocido y aceptado en la bibliografía, es que la frecuencia de ambas entidades aumenta con la edad y este aumento es más acusado en mujeres mayores de 60 años (hasta el 16\% pueden presentar un hipotiroidismo subclínico) (4).

En la gran mayoría de los casos (hasta el 99\%) el hipotiroidismo (manifiesto o subclínico) se produce por una lesión intrínseca del tiroides, siendo la causa más frecuente la tiroiditis crónica autoinmune, definida por la presencia de anticuerpos tiroideos y que tiene dos formas clínicas: una que cursa con bocio, denominada enfermedad de Hashimoto y otra que se acompaña de disminución del tamaño glandular llamada atrofia tiroidea autoinmune. Después se situarían el hipotiroidismo yatrógeno (postquirúrgico o post-radioyodo, que pueden suponer hasta $1 / 3$ de los casos de hipotiroidismo en la comunidad) y con menor frecuencia algunas formas de tiroiditis subagudas, el hipotiroidismo por deficiencia de yodo o por fármacos y el hipotiroidismo congénito.

En la mayor parte de los casos las manifestaciones funcionales, si existen, pueden ser atribuidas a otras etiologías como envejecimiento, menopausia, depresión, etc..., de forma que el paciente hipotiroideo puede ser tratado de manera sintomática y durante largo tiempo por cuadros no específicos: metabólicos (hipercolesterolemia), cardiovasculares (bradicardia, trastornos de conducción), psiquiátricos (depresión, trastornos bipolares), funcionales (astenia, cuadros digestivos o ginecológicos) (2). En la bibliografía revisada, no son muchos los estudios relativos a la valoración económica del hipotiroidismo como entidad aislada, relacionándolo en unos casos con los niveles de colesterol en suero, en otros con la necesidad de hacer pruebas de screening o no y en la mayoría, con el controvertido tema de tratar o no el hipotiroidismo subclínico.

El objetivo del presente estudio es triple:

1. Analizar las manifestaciones clínicas, bioquímicas y epidemiológicas de debut en pacientes con hipotiroidismo. Analizar las posibles diferencias según la causa responsable del hipotiroidismo.

2. Analizar la dosis de hormona tiroidea diaria que reciben, ajustada por peso.

3. Estudiar los costes directos que generan en una consulta especializada anualmente (fármacos, analítica, consulta médica).

\section{MATERIAL Y MÉTODOS}

Se revisaron las historias clínicas de 75 pacientes diagnosticados de hipotiroidismo y seguidos en la consulta de Endocrinología de un área de salud (muestreo no probabilístico continuo). Los datos estudiados se referían al momento del diagnóstico de la enfermedad y se clasificaron en tres tipos:
1. Variables epidemiológicas: nombre, número de historia, edad, sexo, patología tiroidea, patología intercurrente, peso, talla, IMC (índice de masa corporal), tensión arterial sistólica y diastólica.

2. Variables bioquímicas y clínicas: glucosa, creatinina, sodio, potasio, colesterol, LDL colesterol, HDL colesterol, triglicéridos, T4 libre, TSH, anticuerpos antitiroglobulina (ACTG), anticuerpos antiperoxidasa (ACTPO), grado de bocio (palpación tiroidea), dosis de T4 necesaria para eutiroidismo y nombre comercial de la misma, y otros fármacos usados por el paciente.

3. Variables económicas: coste anual en tratamiento sustitutivo con T4, coste anual en analítica (bioquímica general incluyendo hormonas tiroideas, perfil lipídico, hemograma), coste anual en consulta médica.

Las variables epidemiológicas son datos simples que se obtienen de forma sencilla a través de las historias clínicas de cada paciente y referidos al momento del diagnostico, no precisando consideraciones adicionales.

Las variables bioquímicas se obtienen de los resultados hallados en las respectivas analíticas realizadas a los pacientes que son enviados a la consulta de Endocrinología con la sospecha de hipotiroidismo y que confirmaron en su caso el diagnóstico y/o la etiología. A continuación expresamos los diferentes métodos bioquímicos utilizados:

-Bioquímica general: se determinaron los siguientes parámetros mediante un auto analizador (Hitachi, ATM) y con los valores normales que se expresan a continuación: glucosa $(75-110 \mathrm{mg} / \mathrm{dl})$, creatinina $(0,6-1,1 \mathrm{mg} / \mathrm{dl})$, sodio $(135-$ $145 \mathrm{meq} / \mathrm{l})$, potasio $(3,5-4,5 \mathrm{meq} / \mathrm{l})$.

-Perfil lipídico: las concentraciones de colesterol total (110-240 mg/dl) en suero y de triglicéridos (50-170 mg/dl) fueron determinadas por estudio enzimático colorimétrico (Techni con Instruments, Ltd., N.Y., USA), mientras que HDL colesterol $(40-70 \mathrm{mg} / \mathrm{dl})$ fue determinado enzimáticamente en el líquido sobrenadante después de la precipitación de otras lipoproteínas con dextrano sulfato-magnesio y LDL colesterol (85$175 \mathrm{mg} / \mathrm{dl}$ ) fue calculado usando la fórmula de Friedewald.

-Perfil tiroideo: Las concentraciones de T4 libre $(0,70$ $1,48 \mathrm{ng} / \mathrm{dl})$ y de TSH $(0,35-4,94 \mathrm{mUI} / \mathrm{ml})$ se determinaron por inmunoensayo (Abbott-Architec T4 libre, I. L., USA y Abbott-Architec TSH, I. L.,USA, respectivamente). Los anticuerpos antitiroglobulina (ACTG) y antiperoxidasa (ACTPO) fueron determinados por método ELISA mediante Biochem Imnunosistem ElAgen Anti-Tiroglobulina, Bologna, Italia y Biochem Inmunosistem ElAgen Anti-TPO, Bologna, Italia, con los siguientes valores de referencia:

$\begin{array}{lccc} & \text { Negativo } & \text { Dudoso } & \text { Positivo } \\ \text { Anti-tiroglobulina } & <225 \mathrm{UI} / \mathrm{ml} & 225-325 \mathrm{UI} / \mathrm{ml} & >325 \mathrm{UI} / \mathrm{ml} \\ \text { Anti-TPO } & <35 \mathrm{UI} / \mathrm{ml} & 35-50 \mathrm{UI} / \mathrm{ml} & >50 \mathrm{UI} / \mathrm{ml}\end{array}$

Las variables clínicas se refieren al grado de bocio, a la dosis y nombre comercial de la hormona T4 usada como terapia sustitutiva y otras posibles medicaciones usadas por el paciente por patologías intercurrentes. En cuanto al grado de bocio se determina habitualmente por palpación usando la clasificación clínica de la OMS (6):

Grado $0 \rightarrow$ Ausencia de bocio. Tiroides no palpable o palpable pero de tamaño normal.

Grado IA $\rightarrow$ Bocio detectable por palpación y no visible incluso con el cuello en extensión. 
Grado IB $\rightarrow$ Bocio palpable y visible sólo en extensión. mal.

Grado II $\rightarrow$ Bocio visible con el cuello en posición nor-

Grado III $\rightarrow$ Bocio voluminoso, identificable a distancia.

La dosis de T4 se refiere aquella con la que a lo largo del seguimiento del paciente, se consigue que éste se mantenga asintomático clínicamente y su perfil tiroideo analítico esté dentro de los límites normales (TSH normal). Por lo tanto es un dato recogido de la historia clínica según su evolución clínica y analítica reflejada en la misma, una vez conseguida la estabilidad del paciente, y se expresa en $\mu \mathrm{g} /$ día (tal y como vienen dosificadas las formas comerciales existentes en España e incluidas en el Vademécum Internacional).

También se recogen los datos referentes a otros fármacos que reciben los pacientes para cualquier otra patología que presenten.

Las variables económicas incluyen todos los posibles costes derivados del seguimiento del paciente en la consulta de Endocrinología, así como el coste del tratamiento sustitutivo con T4, todo ello durante un año:

- Coste anual en analítica: se recoge el número de analíticas realizadas durante un año en cada paciente, diferenciando si se trata de hemograma, bioquímica general, perfil lipídico y perfil tiroideo, calculando el coste respectivo anual de cada prueba multiplicando el número realizado de cada una por el coste de la misma según los datos proporcionados por el Laboratorio de referencia (Hospital Río Hortega de Valladolid) y que son: hemograma $3 €$; bioquímica general (incluyendo perfil tiroideo) $1,80 €$; perfil lipídico 1,80 €; Ac. antitiroideos $1,80 €$

- Coste anual en consultas: se recogen el número de visitas realizadas por cada paciente a la consulta de Endocrinología en un año y se calcula su coste anual multiplicando dicho número por el precio establecido para cada visita según los datos del contrato-programa para el año 2000 del Hospital Río Hortega de Valladolid y que se estima en $60 €$ consulta.

- Coste anual en tratamiento con T4: se calcula según la dosis de mantenimiento para cada paciente (en $\mathrm{mg} /$ día) y teniendo en cuenta el precio del envase del preparado comercial según figura en la edición del año 2000 del Vademécum Internacional (envase de 50 comprimidos con $50 \mathrm{mg}$ cada uno, $2,06 €)$.

- Coste total: Viene dado por la suma de los costes anuales anteriormente referidos, obteniendo una valoración económica de lo que supone el hipotiroidismo en el área de salud oeste de Valladolid.

\section{ANÁLISIS ESTADÍSTICO}

Con los datos obtenidos se generó una base de datos con el paquete estadístico (SPSS, Inc., II., USA). Se realizó un análisis descriptivo de las variables cuantitativas, expresadas como (media \pm desviación estándar), estos parámetros se compararon mediante el test de la t de Student para variables independientes en las paramétricas y el test de Friedman para no paramétricas. El análisis de la distribución normal de las variables se realizó mediante el test de Kolmogorov-Smirnov. Para realizar la correlación entre variables cuantitativas se utilizó el test de correlación de Spearman. Se consideró un nivel estadísticamente significativo $(\mathrm{p}<0,05)$. Las variables cualitativas se expresan en función de frecuencias (porcentajes).

\section{RESULTADOS}

Se revisaron un total de 75 historias clínicas correspondientes a pacientes diagnosticados de hipotiroidismo y seguidos en la consulta de Endocrinología del área de salud oeste de Valladolid. De ellos, 4 (5,3\%) eran varones y $71(94,7 \%)$ eran mujeres, con una media de edad de 52,07 $\pm 16,58$ años.

La patología responsable del hipotiroidismo en los 75 casos era la siguiente: 36 pacientes $(48,0 \%)$ presentaban tiroiditis de Hashimoto; 35 pacientes $(46,6 \%)$ fueron diagnosticados de tiroiditis autoinmune atrófica; 2 pacientes $(2,7 \%)$ habían recibido radioyodo y otros 2 pacientes habían sufrido una tiroidectomía total. Destaca la mayoría de casos de la tiroiditis crónica autoinmune en sus dos formas clínicas.

Los datos relativos al resto de variables epidemiológicas estudiadas (peso, talla, IMC, tensión arterial sistólica y diastólica) se exponen en la tabla I. Cabe señalar el IMC medio, que situaría nuestra muestra en un grado I de obesidad y también el hecho de que tanto la TAS y la TAD se mantengan dentro de los límites normales.

TABLA I

\begin{tabular}{lc}
\multicolumn{2}{c}{ TABLA I } \\
\multicolumn{1}{c}{ DISTRIBUCIÓ N VARIABLES EPIDEM IO LÓ GICAS } \\
\hline Variable & M edia (Desv. estándar) \\
\hline Talla $(\mathrm{cm})$ & $158 \pm 7$ \\
Peso $(\mathrm{Kg})$ & $68,90 \pm 11,66$ \\
IM C $\left(\mathrm{Kg} / \mathrm{m}^{2}\right)$ & $28,08 \pm 5,28$ \\
TAS $(\mathrm{mmHg})$ & $139,12 \pm 17,04$ \\
TAD $(\mathrm{mmHg})$ & $81,76 \pm 11,26$ \\
\hline
\end{tabular}

IM C: índice masa corporal; TAS: tensión arterial sistólica; TAD: tensión arterial diastólica.

En la tabla II se muestran los resultados de las variables bioquímicas: bioquímica general, perfil lipídico y perfil tiroideo, con sus correspondientes medias y desviación standard. Como es de esperar, la TSH alcanza una media por encima de sus valores de referencia, lo cual define el hipotiroidismo, y

TABLA ॥

\begin{tabular}{|c|c|}
\hline \multicolumn{2}{|c|}{ DISTRIBU CIÓ N VARIABLES BIO Q UÍM ICAS } \\
\hline Variable & Media (Desv. estándar) \\
\hline $\begin{array}{l}\text { Glucosa }(\mathrm{mg} / \mathrm{dl}) \\
\text { Creatinina }(\mathrm{mg} / \mathrm{dl}) \\
\text { Sodio (meq/l) } \\
\text { Potasio (meq/l) } \\
\text { Colesterol total (mg/ dl) } \\
\text { LDL colesterol }(\mathrm{mg} / \mathrm{dl}) \\
\text { HDL colesterol (mg/dl) } \\
\text { Triglicéridos (mg/ dl) } \\
\text { T4 libre }(\mathrm{ng} / \mathrm{dl}) \\
\text { TSH (mUl/ml) } \\
\text { ACTPO }(\mathrm{ml} / \mathrm{ml}) \\
\text { ACTG (Ul/ml) }\end{array}$ & $\begin{array}{c}94,59 \pm 14,83 \\
0,96 \pm 0,17 \\
140,28 \pm 1,74 \\
4,33 \pm 0,18 \\
217,44 \pm 56,21 \\
140,28 \pm 46,04 \\
53,87 \pm 13,04 \\
107,95 \pm 67,80 \\
0,86 \pm 0,28 \\
17,57 \pm 40,14 \\
629,30 \pm 1.049,14 \\
532,11 \pm 1.028,34\end{array}$ \\
\hline
\end{tabular}

ACTPO: anticuerpos antiperoxidasa; ACTG: anticuerpos antitiroglobulina. 
los niveles de Ac tiroideos están francamente elevados como corresponde a la etiología predominante (tiroiditis autoinmune). Considerando los casos de hipotiroidismo subclínico (TSH elevada y T4 libre normal), encontramos 24 pacientes con esta alteración analítica (32\%), y de ellos sólo 9 tenían una $\mathrm{TSH}>10 \mathrm{mUI} / \mathrm{ml}$.

En cuanto al grado de bocio que presentaban en el momento inicial de la enfermedad, se distribuía así: grado 0 , 31 pacientes $(41,3 \%)$; grado Ia, 28 pacientes $(37,3 \%)$; grado Ib, 14 pacientes $(18,7 \%)$; grado II, 1 paciente $(1,3 \%)$; grado III, 1 paciente $(1,3 \%)$. Vemos por tanto que el $58,7 \%$ presentan algún grado de bocio.

De los 63 pacientes que estaban en tratamiento sustitutivo con T4, $58(77,3 \%)$ lo hacían con el preparado comercial Levothroid $^{\circledR}$, y $5(6,7 \%)$ con el preparado Dexnon ${ }^{\circledR}$. Los 12 pacientes restantes $(16 \%)$ no precisaban tratamiento. La dosis media necesaria para el correcto control de los pacientes fue de $81,00 \pm 53,56 \mathrm{mg} /$ día.

La media de visitas anuales a la consulta de Endocrinología que precisaron los pacientes hasta alcanzar la normofunción tiroidea fue de 2,67 $\pm 1,08$. El número de pruebas analíticas realizadas en un año fueron muy similares para todas ellas (media \pm desv. estándar): hemograma, 1,95 $\pm 0,87$; bioquímica general, 1,96 $\pm 0,86$; perfil lipídico, 1,97 $\pm 0,89$; perfil tiroideo, $1,97 \pm 0,87$. Estos datos muestran que las pruebas analíticas se piden a la vez, aprovechando un control para realizar toda la batería de pruebas.

Los resultados en costes de los datos generales previos según lo explicado en el apartado de material y métodos, se exponen en la tabla III. Como sería de esperar, el mayor coste corresponde a la consulta especializada, tanto por un mayor número de visitas realizadas, como por un mayor coste propio. En cambio, el gasto en tratamiento sustitutivo con T4 es relativamente bajo, sin duda por el también bajo precio de la forma farmacéutica y la baja dosis necesaria para un buen control.

A la vista de la prevalencia en el grupo estudiado de la etiología autoinmune, en un segundo tiempo se hace un estudio comparativo de las distintas variables en la tiroiditis de Hashimoto y la tiroiditis autoinmune atrófica, analizando si existe alguna significación estadística en los resultados obtenidos. Dichos resultados se exponen en la tabla IV. Como podemos comprobar los costes fueron superiores en los pacientes con tiroiditis de Hashimoto, probablemente en relación con el mayor número de visitas que precisaron para alcanzar la normofunción tiroidea y también la dosis de T4 que requerían fue superior, aunque no estadísticamente significativa.

\section{TABLA III}

DISTRIBUCIÓ N COSTE DIRECTO ANUAL DE LAS DISTINTAS

VARIABLES ESTUDIADAS, INCLUYENDO EL COSTE TOTAL GENERADO EN UNA CONSULTA ESPECIALIZADA

\begin{tabular}{lc}
\hline Variable & M edia (Desv. estándar) \\
\hline Coste consulta (ptas./año) & $26.666,67 \pm 10.822,07$ \\
Coste hemograma (ptas./año) & $1.946,67 \pm 868,26$ \\
Coste bioquímica y perfil tiroideo & $1.066,67 \pm 432,88$ \\
$\quad$ (ptas./año) & $966,67 \pm 445,19$ \\
Coste perfil lipídico (ptas./año) & $1.073,97 \pm 431,41$ \\
Coste trato. T4 (ptas./año) & $354.26,37 \pm 13.564,95$ \\
Coste total (ptas./año) & \\
\hline
\end{tabular}

\section{DISCUSIÓN}

El hipotiroidismo es un frecuente motivo de consulta en Endocrinología, bien por sospecha clínica del médico de Atención Primaria o, la mayoría de las ocasiones, tras un hallazgo casual en análisis realizados por otros motivos. La confirmación de dicha sospecha conlleva la realización una serie de pruebas analíticas para verificar el diagnóstico y, si es posible, su etiología y posteriormente, obliga a un seguimiento del paciente para controlar su evolución y tratamiento. Por todo ello, el presente estudio, aparte de las características clínicas y analíticas, trata de describir el gasto que supone al año un caso de hipotiroidismo desde que se realice el diagnóstico.

Desde el punto de vista clínico y epidemiológico, el hipotiroidismo es un tema ampliamente estudiado y revisado en la literatura. Es conocida su mayor prevalencia en mujeres, y en nuestro caso así resulta con un $94,7 \%$ de mujeres, aunque no es un estudio equiparable a otros realizados, ni por el muestreo ni por la población estudiada. T. Bjoro y cols. (7), en el estudio de Nord-Trondelag (Noruega), obtienen una prevalencia del $4,8 \%$ en mujeres y $0,9 \%$ en hombres para el hipotiroidismo en la población general (5:1), mientras que Vanderpump y cols. (8), en el Wickham Survey, ofrecieron datos de un 7,1 y $0,5 \%$ respectivamente. Igualmente admitido es que la prevalencia aumenta con la edad, con un límite que los diversos estudios sitúan a partir de los 50 años, siendo más acusado este aumento en las mujeres; en nuestro estudio la media de edad se sitúa en 52 años, lo cuál estaría dentro de lo esperado.

En referencia a los datos epidemiológicos pudimos observar como el IMC, con una media de 28,08 puede hacer pensar en una tendencia de la enfermedad a la obesidad ligera, cosa por otra parte lógica si tenemos en cuenta la fisiopatología del hipotiroidismo.

Clínicamente, la presencia de bocio registrada en nuestro estudio fue del $58,7 \%$, pero sólo en el $2,6 \%$ podemos hablar de un grado de bocio evidente (grados II y III). Esto nos llevaría a pensar en una relativamente baja frecuencia de este signo en el hipotiroidismo. En el estudio de Nord-Trondelag (Noruega) (7), se refieren al bocio autoreferido con una prevalencia del $2,9 \%$ en mujeres y del $0,4 \%$ en hombres, aumentando con la edad en ambos.

Por lo que respecta a la causa o enfermedad responsable del hipotiroidismo, hay concordancia con lo admitido en la literatura, siendo la etiología autoinmune la más frecuente, tanto en nuestro estudio como en la bibliografía revisada $(9,10)$. Como es evidente, hay una relación directa con la presencia o no de anticuerpos tiroideos, tema que será analizado más adelante.

Desde el punto de vista analítico, hay que distinguir varios aspectos. En primer lugar, los datos de la bioquímica general (glucosa, $\mathrm{Na}, \mathrm{K}$, creatinina) no parecen aportar ninguna característica relevante en nuestro estudio, con valores medios dentro de la normalidad. En cuanto al perfil lipídico, los valores medios obtenidos en nuestro estudio tanto de colesterol total, LDL, HDL y triglicéridos están dentro de los rangos de referencia, lo que podría inducirnos a pensar que no hay efectos metabólicos en el hipotiroidismo, cuándo es sobradamente conocido lo contrario a la vista de su fisiopatología, y aún más cuándo en la mayoría de los estudios sobre screening del fallo tiroideo se utiliza el perfil lipídico como factor de despistaje. Estos resultados coincidirían con los de T. Bjoro y cols., pues no encuentran diferencias en el perfil lipídico en personas con 
TABLA IV

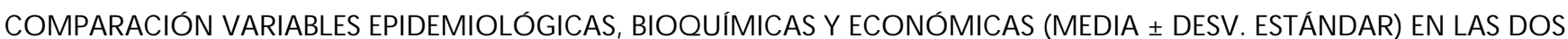
FO RM AS ETIO LÓ GICAS M ÁS PREV ALENTES, T. DE HASHIM OTO Y T. AUTO INM UNE ATRÓ FICA

\begin{tabular}{|c|c|c|c|}
\hline$\overline{\text { Variable }}$ & T. de Hashimoto & T. autoinmune atrófica & \\
\hline$\overline{\text { Edad (años) }}$ & $48,66 \pm 16,64$ & $56,22 \pm 16,41$ & \\
\hline IMC & $27,49 \pm 5,7$ & $27,98 \pm 4,25$ & \\
\hline TAS (mmHg) & $141,66 \pm 18,69$ & $136,61 \pm 15,24$ & \\
\hline TAD (mmHg) & $81,51 \pm 10,19$ & $82,90 \pm 11,95$ & \\
\hline Glucosa (mg/dl) & $95,75 \pm 15,84$ & $92,54 \pm 13,77$ & \\
\hline $\mathrm{Na}(\mathrm{meq} / \mathrm{l})$ & $139,91 \pm 1,68$ & $140,88 \pm 1,51$ & $(* p=0,01)$ \\
\hline $\mathrm{K}(\mathrm{meq} / \mathrm{l})$ & $4,28 \pm 0,16$ & $4,36 \pm 0,18$ & \\
\hline Creatinina (mg/dl) & $0,93 \pm 0,16$ & $0,97 \pm 0,18$ & \\
\hline Colesterol (mg/ dl) & $208,13 \pm 46,82$ & $224,65 \pm 65,31$ & \\
\hline LDL Colesterol (mg/ dl) & $133,88 \pm 38,56$ & $146,08 \pm 54,13$ & \\
\hline $\mathrm{HDL}$ colesterol (mg/dl) & $53,36 \pm 14,47$ & $53,65 \pm 11,62$ & \\
\hline Triglicéridos (mg/dl) & $101,80 \pm 44,95$ & $116,42 \pm 87,86$ & \\
\hline T4 libre (ng/dl) & $0,86 \pm 0,28$ & $0,84 \pm 0,27$ & \\
\hline TSH (mUl/ml) & $13,93 \pm 15,67$ & $13,06 \pm 16,76$ & \\
\hline ACTG (Ul/ml) & $665,82 \pm 1.110,93$ & $438,51 \pm 994,87$ & \\
\hline ACTPO (UI/ml) & $975,40 \pm 1.172,02$ & $210,54 \pm 301,92$ & $(* p=0,00)$ \\
\hline Dosis T4 (mq/ día) & $92,36 \pm 54,05$ & $70,00 \pm 51,01$ & \\
\hline Visitas anuales & $2,97 \pm 1,02$ & $2,40 \pm 1,09$ & $(* p=0,02)$ \\
\hline Coste visitas (ptas./año) & $29.722,22 \pm 10.277,88$ & $24.000,00 \pm 10.900,62$ & $(* p=0,02)$ \\
\hline Coste hemograma (ptas.año) & $2.111,11 \pm 887,30$ & $1.800,00 \pm 833,13$ & \\
\hline $\begin{array}{l}\text { Coste bioquímica y } \\
\text { perfil tiroideo (ptas./ año) }\end{array}$ & $1.188,88 \pm 411,11$ & $960,00 \pm 436,02$ & $(* p=0,02)$ \\
\hline Coste perfil lipídico (ptas./año) & $1.069,44 \pm 449,64$ & $871,42 \pm 426,04$ & \\
\hline Coste T4 (ptas./año) & $1.188,88 \pm 411,11$ & $952,94 \pm 440,54$ & $(* p=0,02)$ \\
\hline Coste Total (ptas./ año) & $39.514,38 \pm 13.028,91$ & $31.800,22 \pm 13.404,96$ & $(* p=0,01)$ \\
\hline
\end{tabular}

$(*)$ : M edidas con significación estadística.

hipotiroidismo subclínico respecto al grupo control, pero cuando los niveles de TSH son mayores de $10 \mathrm{mU} / \mathrm{l}$, si hay incrementos de colesterol total y LDL y descensos de HDL. De aquí se podría afirmar la relativa importancia del $32 \%$ de hipotiroidismo subclínico hallado en nuestro estudio. En un tercer apartado, el perfil tiroideo nos da un valor medio de T4 libre de 0,86 ng/dl y de TSH de 17,57 mUI/ml. Estas cifras corroboran lo afirmado anteriormente sobre la importancia del hipotiroidismo subclínico en nuestro estudio y, aunque no son comparables con otros estudios, si se puede ver cierto paralelismo con los datos del estudio de Nord-Trondelag (7) (Noruega) de T. Bjoro y cols., que obtiene una prevalencia de TSH> $10 \mathrm{mU} / 1$ del $0,37 \%$ en hombres y del $0,9 \%$ en mujeres. Además, hay otro dato de interés como es el que relaciona los niveles de TSH con los de T4 libre: sólo el 37\% de hombres y el $56 \%$ de mujeres con TSH> 10 mU/l tenían también una T4 libre disminuida. Es decir, no siempre hay una correlación entre los niveles de ambos parámetros. Un último apartado es el relativo a los anticuerpos tiroideos, con una media de ACTPO de 629,30 UI/ml y de ACTG de 532,11 UI $/ \mathrm{ml}$, ambos por encima del rango de referencia, más acusado en el caso de los ACTPO, y ello enlazaría con la mayor prevalencia de la etiología autoinmune y confirma la importancia de la autoinmunidad en el hipotiroidismo. En el ya mencionado estudio de Nord-Trondelag (Noruega) (7), sólo ofrecen datos de ACTPO, que está presente en un $2,8 \%$ de hombres y un $13,9 \%$ de mujeres mayores de 40 años y en relación con los niveles de
TSH y la edad, encuentran que es más precoz el hallazgo de anticuerpos que el aumento de TSH. El mayor aumento de los ACTPO iría a favor de su mayor especificidad respecto a los desordenes tiroideos y en particular a la etiología autoinmune.

Por lo que se refiere a la dosis de mantenimiento de T4 requerida por los pacientes de nuestro estudio, obtenemos una media de $81,0 \mathrm{mg} /$ día, que como se comentó anteriormente se puede considerar baja. En el estudio de Nord-Trondelag (Noruega) (7), según datos de 1997, la dosis media diaria de tiroxina se sitúa entre 100 y $125 \mathrm{mg}$, cifras mayores a las de nuestro estudio. Del apartado relativo al preparado comercial de tiroxina habitualmente usado, la gran diferencia de Levothroid $^{\circledR}$ no precisa más comentario que sus dos presentaciones, $50 \mathrm{mg}$ y $100 \mathrm{mg}$, facilitan el ajuste de dosis.

Pasando al apartado económico del estudio, hay varios elementos que comentar. Uno de ellos se refiere al número de visitas anuales que precisan los pacientes, cuya media fue de 2,67 visitas al año, lo que se traduciría en una visita a la consulta de Endocrinología cada 4 meses y medio, aproximadamente. En cualquier caso esta cifra es difícil de valorar, pues el distinto comportamiento farmacocinético de la tiroxina en cada paciente hace que el adecuado control clínico y bioquímico sea más o menos difícil en cada caso y eso pueda reflejarse en un mayor número de controles y por tanto de consultas al especialista. Además, esa misma farmacocinética dificulta en ocasiones hallar la dosis precisa para el correcto control del paciente, con un umbral muy sensible que puede 
hacer que con mínimos aumentos o descensos de dosis nos pasemos o nos quedemos cortos. En concordancia con el número de visitas se sitúa el número de las respectivas pruebas analíticas, que, como se comentó en su momento, coinciden con cada consulta al endocrino salvo en la primera que se dedica básicamente a la anamnesis y exploración, ya que la analítica inicial proviene en la mayoría de los caso de Atención Primaria.

Como era de esperar el mayor coste corresponde a la consulta especializada, con una media de 160,26 € al año, mientras que los costes de hemograma $(11,69 € /$ año), bioquímica y perfil tiroideo $(6,40 € /$ año) y perfil lipídico $(5,80$ $€ /$ año) se sitúan a mucha distancia. También el gasto que representa el tratamiento sustitutivo de T4 (6,44 €/año) se puede considerar poco relevante por el bajo precio de las formas farmacológicas. En un estudio en 1988 de X. Lenne y cols. (2). sobre "evaluación económica del diagnostico y tratamiento del hipotiroidismo ante una hipercolesterolemia", se ofrece un coste diario del tratamiento sustitutivo de $0,09 €$, no comparable con nuestro estudio pues se calcula en base a una dosis media de $100-150 \mathrm{mg} /$ día. Este punto del coste del tratamiento con tiroxina relacionado con los beneficios clínicos y analíticos resultantes del mismo, es argumentado en la literatura revisada como un punto a favor del screening y tratamiento del hipotiroidismo subclínico, evitándose su posible progresión a hipotiroidismo manifiesto. El coste total medio resultante de todo lo anterior es de $212,9 €$ al año para cada paciente, cifra que por sí sola no dice mucho, pero considerando globalmente el resultado del gasto que supone el diagnóstico y seguimiento de todos los pacientes de esta patología tan prevalente, estaríamos ante una cifra respetable y más en los tiempos actuales en que el gasto sanitario parece imparable y los recursos del sistema son limitados, tendiéndose a medir todo en términos costeefectividad.

En el estudio referido de X. Lenne y cols. (2). se recogen algunos datos económicos, expresados en francos de 1988, no equiparables a los de nuestro estudio pues parten de un diagnóstico previo de hipercolesterolemia para posteriormente realizar el de hipotiroidismo. Obtienen un coste medio del diagnóstico inicial de hipotiroidismo (incluyendo la consulta médica y la dosificación de TSH y/o T4) de 46,44 €. En el caso de un hipotiroidismo manifiesto establecen 2 a 4 consultas al generalista anuales con un coste de $17,52 €$ cada una, y cuatro controles analíticos de TSH durante el primer año con un coste de 19,20€ cada uno. En el caso de un hipotiroidismo subclínico calculan dos consultas al año al generalista $(17,5 €)$ y una determinación de TSH + T4 con un coste de 94,76 €. A esto habría que añadir lo comentado anteriormente sobre el coste del tratamiento sustitutivo con $\mathrm{T} 4$.

En otro estudio de D. Danese y cols. (11) sobre "análisis del screening para enfermedad tiroidea leve", se muestran los siguientes costes en dólares (1.994): determinación TSH en suero, $25 \$(27,90 €)$; determinación T4 libre, $14 \$(15,60 €)$; título de anticuerpos microsomales, $22 \$(24,6 €)$; visitas de seguimiento, $24 \$(26,8 €)$; coste anual de terapia con tiroxina (media de $125 \mathrm{mg}$ ), $62 \$(69,35 €)$. Como vemos, cada estudio tiene costes y resultados muy variables, según su diseño y finalidad, lo que hace difícil su comparación con nuestro estudio.

Pasando al estudio comparativo entre las dos etiologías autoinmunes predominantes, comprobamos en primer lugar que no existen diferencias significativas en las variables epidemiológicas estudiadas (edad, IMC y tensión arterial), como era de esperar. Tampoco los resultados de bioquímica general ni el perfil lipídico muestran diferencias significativas, cosa también esperada en un principio. Sólo destacar la excepción de los niveles de sodio, cuya interpretación no va más allá probablemente de unos valores con mínima dispersión respecto a la media. En el perfil tiroideo, hay un mayor nivel de anticuerpos en la tiroiditis de Hashimoto, pero sólo los resultados de ACTPO son significativos, lo que se explicaría por la mayor especificidad de estos anticuerpos en la tiroiditis de Hashimoto, que, en general presenta mayores niveles de anticuerpos que la tiroiditis autoinmune atrófica. También se observa una mayor necesidad de dosis sustitutiva de T4 en la tiroiditis de Hashimoto, pero sin llegar a tener significación estadística. Donde se obtienen las diferencias más significativas es en el análisis de las variables económicas. Así tenemos un número de visitas anuales mayor en la tiroiditis de Hashimoto, con un mayor coste anual de estas visitas; en cambio, de los costes analíticos, solamente es significativo el dato de la bioquímica y perfil tiroideo. Igualmente resulta significativo el coste en terapia sustitutiva con tiroxina, a pesar de que cómo dijimos anteriormente, sus diferencias en dosis necesaria no eran significativas, aunque si con una tendencia a ser superior en pacientes con tiroiditis de Hashimoto. Finalmente, como consecuencia de las diferencias significativas en gran parte de los costes analizados, obtenemos también diferencias significativas en el coste total entre ambas etiologías, lo que implica un mayor requerimiento en recursos en el caso de la tiroiditis de Hashimoto.

El hipotiroidismo representa un frecuente motivo de consulta tanto en Atención Primaria como en Atención Especializada, con un importante gasto de recursos por su creciente prevalencia, en gran parte debido a que contamos con métodos diagnósticos cada vez más simples y al alcance de cualquier nivel asistencial.

Es ampliamente conocida la mayor prevalencia del hipotiroidismo en las mujeres y que aumenta con la edad, sobre todo a partir de los 50 años. No encontramos en nuestro estudio relación con los valores de tensión arterial ni con el índice de masa corporal. Sin duda el test más rentable para el diagnóstico inicial de hipotiroidismo es la determinación en suero de los niveles de TSH y T4 libre. No se demuestra en nuestro estudio la importancia o no de las alteraciones del perfil lipídico. La etiología más frecuente es la de origen autoinmune, comprobándose la especificidad de los ACTPO con la tiroiditis de Hashimoto.

En cuanto al análisis económico, aunque los costes analíticos no son altos, la necesidad de controles periódicos hasta un adecuado control, a veces difícil, hace que el coste total anual de estos pacientes se eleve a cifras moderadamente altas, dificultando la decisión sobre si realizar screening o no a la población general, teniendo en cuenta que aquellos que sean detectados como enfermos entrarán en el círculo de consultas y controles periódicos, con el aumento de costes consiguiente en personas hasta entonces asintomáticas y con pocas probabilidades de complicaciones agudas.

Por último, comprobamos el mayor coste en el caso de tiroiditis de Hashimoto en consultas, analíticas y en tratamiento sustitutivo y, por consiguiente, un mayor coste total, lo cual confirmaría su mayor trascendencia clínica respecto a la tiroiditis atrófica autoinmune. 


\section{Bibliografía}

1. Sanmartí Sala A, Rius Riu F. ¿Tratar o no tratar el hipotiroidismo asintomático o subclínico?. Form Med Atn Prim 1994; 7: 391-2.

2. Lenne X, Allenet B, Laurent P, Lebrun T, Wemeau JL. Evaluation économique de l'intérêt du diagnostic et de la prise en charge precose de l'hypothyroïdie devant une hypercholestérolémie. Ann Endocrinologie (Paris) 1999; 60: 398-407.

3. Bilous RW, Tumbrigde WMG. The epidemiology of hypothyroidism. An update. Baillière's. Clin Endocrinol Metab 1998; 2: 531-40.

4. MI. Surks, E. Ocampo. Subclinical thyroid disease. Am J Med 1996; 100: 217-23.

5. Tumbrigde WM, Evered DC, Hall R, Appelton D, Brewis M, Clark F, et al. The spectrum of thyroid disease in a community: the Wickham survey. Clin Endocrinil 1977; 7: 481-93.

6. Delange F, Bastani S, Benmiloud M, et al. Definitions of endemic goiter and endemic cretinism, classification of goiter size and severity of endemias, and survey techniques. En: JT. Dunn, ed. Towards the erradication of endemic goiter, cretinism and iodine deficiency. Washingtong: PAHO, Scientifics Publication, 1996; 502: 373-6.
7. Bjoro T, Holmen J, Krüger $\varnothing$, Midthjell K, Hunstad K, Schreiner T, et al. Prevalence of thyroid disease, thyroid dysfunction and thyroid peroxidase antibodies in a large, unselected population. The Health Study of Nor-Trondelag (HUNT). Eur J Endocrinol 2000; 143: 63947.

8. Vanderpump MPJ, Tunbridge WMG, French JM, Appleton D, Bates D, Clark F, et al. The incidence of thyroid disorders in the community: a twenty-year follow-up of the Wickham Survey. Clin Endocrinol 1995; 43: $55-68$

9. Dayan CM, Daniels GH. Chronic autoimmune thyroiditis. N Engl J Med 1996; 335: 99-107.

10. TunbridgeWMG, Vanderpump MPJ. Population screening for Autoimmune Thyroid disease. Endocrinol Metabol Clin North Am 2000; 29: 239-53.

11. Danese Neil KD, Powe Clark R, Sawin T, Paul W. Ladenson. Screening for Mild Thyroid Failure at the Periodic Health Examination; A Decision and Cost-effectiveness Analysis. JAMA 1996; 276: 28592. 